\title{
PRIMER REGISTRO DE HIBISCUS TRIONUM (MALVACEAE) PARA ARGENTINA, EN EL ALTO VALLE DE RÍO NEGRO
}

\author{
Carlos R. Minué \& Ricardo Gandullo
}

Departamento de Biología Aplicada, Facultad de Ciencias Agrarias, Universidad Nacional del Comahue, RN $N^{\circ} 151 \mathrm{~km}$ 12,5, Casilla de Correo 85, 8303 Cinco Saltos, Río Negro, Argentina; carlosminue@hotmail.com (autor corresponsal).

\begin{abstract}
Minué, C. R. \& R. Gandullo. 2019. First record of Hibiscus trionum (Malvaceae) for Argentina, in the Alto Valle de Río Negro. Darwiniana, nueva serie 7(1): 167-173.

The naturalization of Hibiscus trionum (Malvaceae), a crop weed widely distributed worldwide, is reported for the first time for Argentina. The species is described on the material collected for this study. In addition, its uses, distribution and habitat, common names and a key to differentiate the species of Hibiscus cited for the Flora of Argentina are included. Two figures with photographs that complement the description of the species are presented.
\end{abstract}

Keywords. Argentinean Flora; Hibiscus; Malvaceae; weed.

Resumen. Minué, C. R. \& R. Gandullo. 2019. Primer registro de Hibiscus trionum (Malvaceae) para Argentina, en el Alto Valle de Río Negro. Darwiniana, nueva serie 7(1): 167-173.

Se cita por primera vez a Hibiscus trionum (Malvaceae), una maleza de cultivos con amplia distribución mundial, naturalizada en la Argentina. La especie se describe a partir del material recolectado para este estudio. Además, se incluyen sus usos, distribución y hábitat, nombres vulgares y una clave para diferenciar las especies de Hibiscus citadas para la Flora Argentina. Se presentan dos figuras con fotografías que complementan la descripción de la especie.

Palabras clave. Flora Argentina; Hibiscus; maleza; Malvaceae.

\section{INTRODUCCIÓN}

En este trabajo se describe y se cita por primera vez a Hibiscus trionum L. como naturalizada para la Flora Argentina, hallada recientemente en el Alto Valle de Río Negro. Este aporte surge en el marco de investigaciones sobre flora y vegetación de suelos salinizados. H. trionum es una especie originaria de Europa y se encuentra citada para Chile y Uruguay (Zuloaga et al., 2019). Es considerada maleza por su alta producción de semillas y una elevada viabilidad y latencia de las mismas, que conlleva a su persistencia en el suelo (Westra et al., 1996; Chachalis et al., 2008; Odero et al., 2009; Walker et al., 2010; Sobrero et al., 2014), en cultivos de manzano, peral, vid, maíz, tomate, entre otros (Rodríguez et al., 1989; Westra et al., 1990; CABI, 2019), típicos de la región del Alto Valle de Río Negro.

La familia Malvaceae presenta una distribución cosmopolita con más de 100 géneros y 2000 especies, originarias de regiones tropicales a templadas del mundo, reunidas en 5 tribus (Paiva, 2005). Dentro de la tribu Hibisceae se 
destaca el género Hibiscus L., que contiene más de 200 especies (Nogueira \& Paiva, 2005), muchas de ellas reconocidas por su interés económico (textil y maderero) y ornamental (Rodrigo, 1948; Winters, 1970; Dimitri, 1972). El Catálogo de la Flora del Cono Sur registra 11 especies, de las cuales 6 están presentes en la Argentina (Zuloaga et al., 2019). La presente contribución aumenta a 7 el número de especies de Hibiscus para este país.

\section{MATERIALES Y MÉTODOS}

El material colectado proviene de relevamientos realizados en diferentes localidades del Alto Valle de Río Negro, durante los años 2015 a 2019. Para la determinación se siguieron los métodos clásicos de taxonomía y se consultó bibliografía nacional (Rodrigo, 1948; Dimitri, 1972; Zuloaga et al., 2019) y extranjera (Nogueira \& Paiva, 2005; Paiva, 2005; Flora of Pakistan, 2019a) relacionada al género Hibiscus. Se contrastó el material con imágenes digitales de alta resolución del lectotipo (BM000646492) de JSTOR Plant Science (http://plants.jstor.org/). Se extrajeron muestras de suelo superficial para evaluar, a campo, su textura. El material de referencia empleado fue depositado en el Herbario Agronomía Región Comahue (ARC) de la Facultad de Ciencias Agrarias, Universidad Nacional del Comahue (Thiers, 2019).

\section{RESULTADOS}

Hibiscus trionum L., Sp. Pl. 2: 697. 1753. TIPO: "Habitat in Italia, Africa" (lectotipo, BM cb 000646492!, designado por Iamonico \& Peruzzi, Taxon 63: 162. 2014). Figs. 1-2.

Hierba anual, de 10-40 $\mathrm{cm}$ de alto. Tallo delgado, erecto, escabroso, con denso indumento de pelos rígidos simples y patentes. Hojas alternas; las basales orbiculares a ovadas con margen aserrado, las medias y superiores profundamente 3-lobadas, lóbulos linearoblongos con margen lobulado, de $2-4 \mathrm{~cm}$, cara abaxial con denso indumento de pelos estrellados; pecíolo de 1-3 cm, hirsuto; estípulas 2, filiformes, de $3 \mathrm{~mm}$. Calículo con 10-12 piezas filiformes, de 0,8-1 cm, connadas en la base, curvadas, híspidas. Botones florales escabrosos, con pelos simples y patentes, de 0,8-1 cm de diám. Flores actinomorfas, de 2-5 $\mathrm{cm}$ de diám., axilares, solitarias; pedicelos de 1-4 cm. Cáliz persistente, de 1-2,5 cm de diám., verdoso, inflado, acrescente en la fructificación; sépalos concrescentes, triangulares con nervios longitudinales violáceos y pilosos. Pétalos de 1,5-2,5 cm, blanco-cremosos, con mácula violácea en la base, contornos lisos, banda violácea longitudinal en la cara abaxial. Estambres numerosos, anteras amarillas. Estigmas 5. Cápsula globosa a subglobosa, hirsuta, ca. 1,3 cm de long. y 1,3 cm de diám. Semillas numerosas, negras, arriñonadas, verrugosas, de 1,6-2 $\mathrm{mm}$.

Iconografía. Curtis (1792: tab. 209); Sibthorp \& Smith (1830: tab. 666); Mohlenbrock (1931: 39, fig. 16); Pammel (1913: 22, fig. 127); Rodríguez et al. (1989: 120).

Nombres vulgares. No se conocen nombres vernáculos de la especie en la Argentina. Algunos nombres en español asignados a la especie corresponden a "aurora", "malva vejigosa" y "malva vesicaria" (Caius, 1942; Nogueira \& Paiva, 2005). En Estados Unidos de América y Australia es conocida como "bladder ketmia", "venice mallow", "flower-of-an-hour" y "shoofly" (Pammel, 1913; Mohlenbrock, 1931; Westra et al., 1996; Cheers, 1999).

Distribución y hábitat. Especie europea, naturalizada en Norteamérica y ampliamente distribuida en África, Asia y Oceanía (Naqshi et al., 1988; CABI, 2019; Flora of Pakistan, 2019b). En Sudamérica, Hibiscus trionum está citada para Chile y Uruguay (Rodríguez et al., 1989; Zuloaga et al., 2019). Esta especie es un terófito que habita ambientes disturbados, como campos cultivados y bordes de caminos (Mohlenbrock, 1931; Ya et al., 2007). En tierras cultivadas requiere suelo desnudo para la germinación y sombra para su desarrollo (CABI, 2019). 

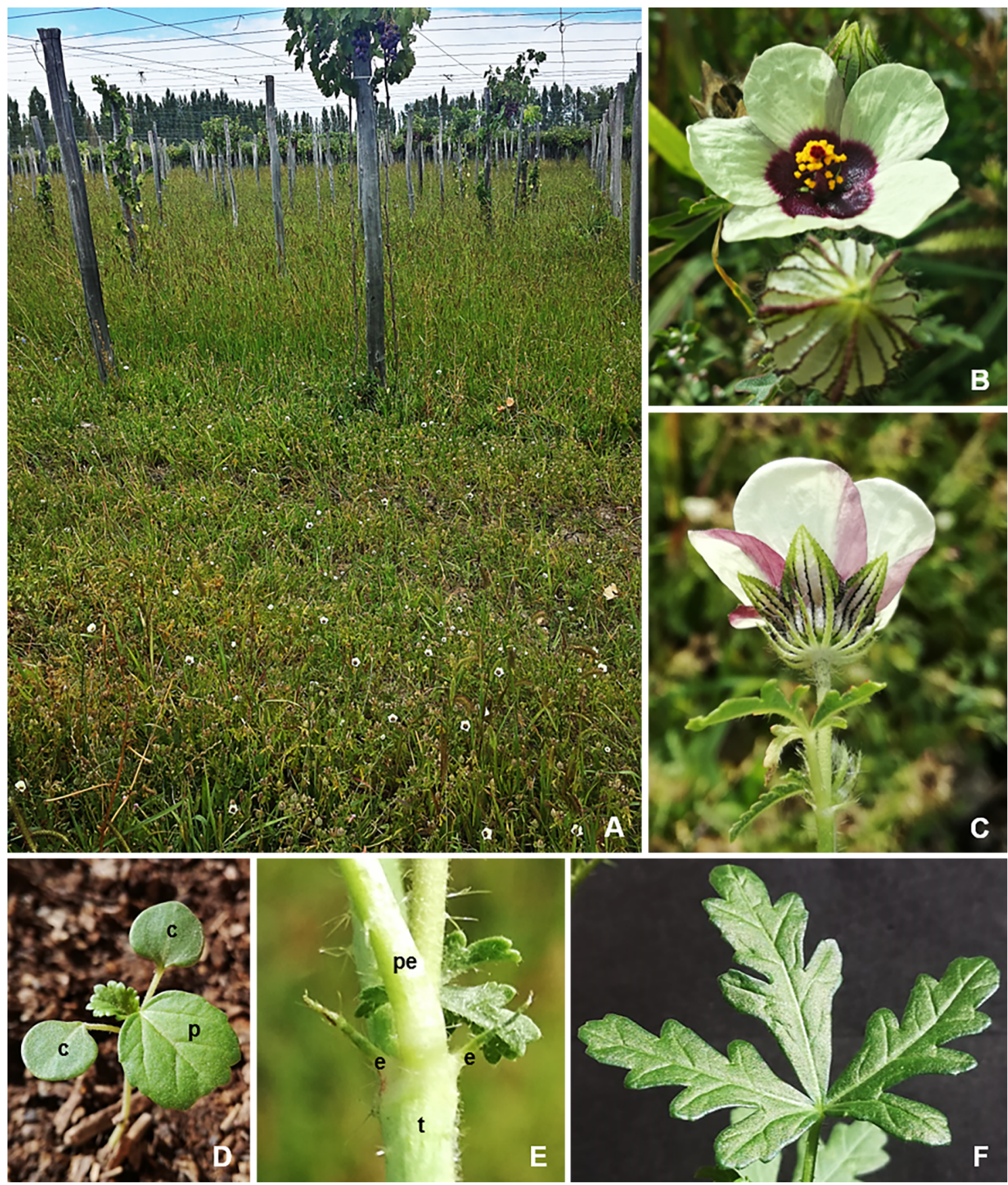

Fig. 1. Hibiscus trionum L. A, vista general de las poblaciones establecidas en el interfilar del cultivo de vid en la localidad de Mainqué, provincia de Río Negro. B, detalle de la flor, nótese la iridiscencia de la mácula central violácea de los pétalos. C, detalle de la parte externa de la flor, nótense las bandas violáceas en el margen de la cara abaxial de los pétalos, la venación oscura en los sépalos y la presencia del calículo. D, detalle de plántula, cotiledones (c) y hoja primordial (p). E, detalle "in vivo" del nudo, véanse las estípulas filiformes pilosas (e) entre el tallo (t) y la base del pecíolo (pe). F, detalle del nomófilo profundamente trilobado. Figura en color en la versión en línea http://www.ojs.darwin.edu.ar/index.php/darwiniana/article/view/829/1153 

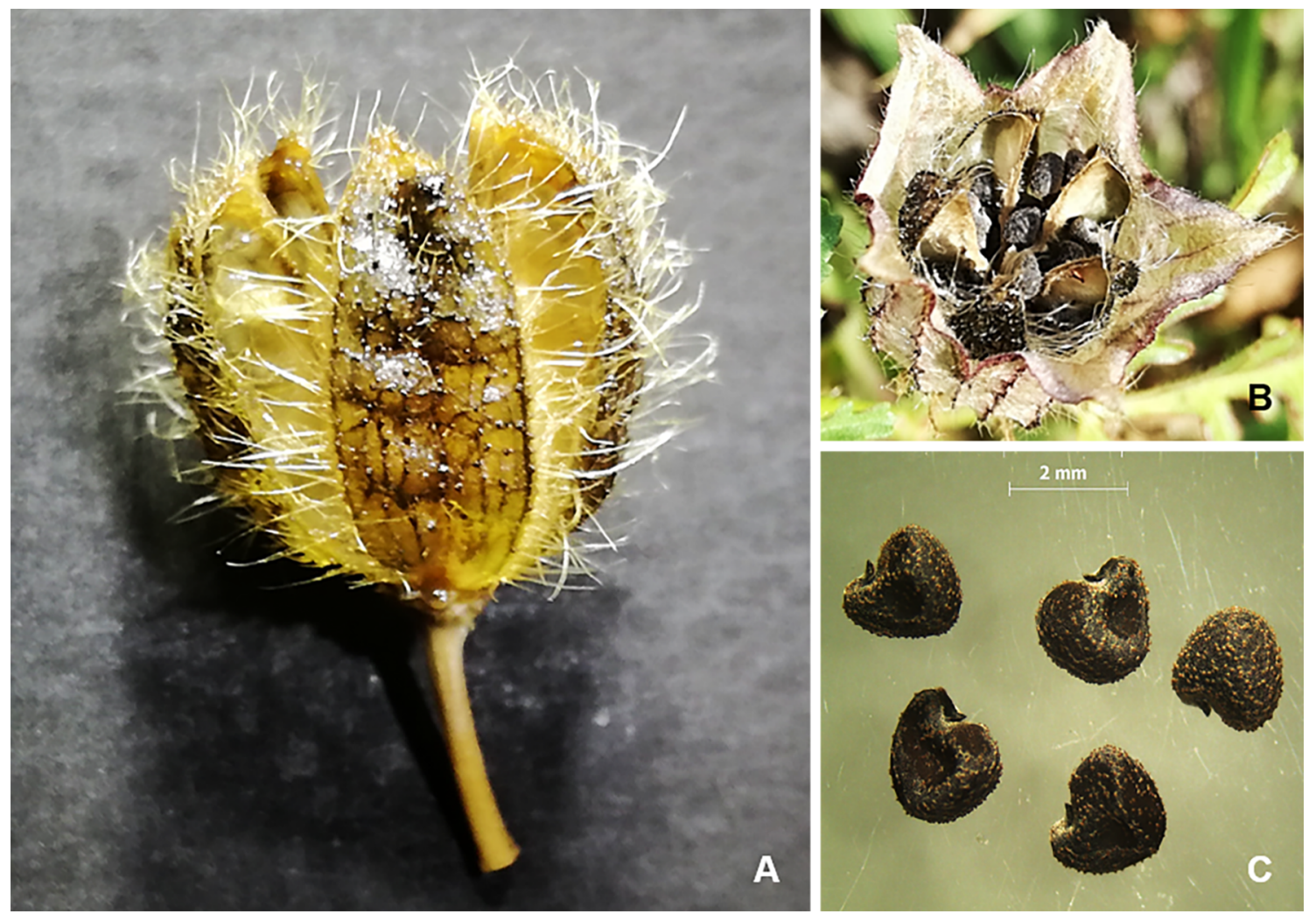

Fig. 2. Hibiscus trionum L. A, vista de la cápsula sin cáliz, nótense los abundantes pelos simples. B, detalle del fruto, nótense el cáliz acrescente, las cinco valvas de la cápsula y semillas. C, detalle de las semillas, nótese la superficie verrugosa. Figura en color en la versión en línea http://www.ojs.darwin.edu.ar/index.php/darwiniana/article/view/829/1153

En el Alto Valle de Río Negro crece como maleza en los cultivos de vid bajo riego, situación coincidente con lo reportado por el Centro de Biociencia Agrícola Internacional (CABI, 2019). De manera similar a lo planteado por Tourn et al. (2018) para otras malezas, el tránsito del tractor junto a sus implementos, en distintas etapas del cultivo, y el riego artificial promueven el desarrollo de amplias y densas poblaciones mediante la dispersión de sus semillas. El área de observación de la especie comprende la localidad de Mainqué, en la provincia de Río Negro (Fig. 3), donde desarrolla densas poblaciones. Comparte el hábitat con Cichorium intybus L., Convolvulus arvensis L., Echinochloa crus-galli var. mitis (Pursh) Peterm., Portulaca oleracea L., Setaria parviflora var. parviflora y Anoda cristata (L.) Schltdl.
Usos. Planta ornamental cultivada en el país (Dimitri, 1972; Martínez Carretero \& Ganci, 2011). Posee una vistosa floración, aunque efímera (Curtis, 1792). Se multiplica por semillas. En Sudáfrica, las plantas se emplean para la elaboración de antihelmínticos, mientras que, en China y Malasia, las hojas secas son destinadas para remedios estomacales (Caius, 1942; Naqshi et al., 1988). La infusión de flores se usa para enfermedades dolorosas de la piel y como diurética (Naqshi et al., 1988). Esta especie se encuentra reportada como tóxica para el ganado, en especial para equinos (Naqshi et al., 1988).

Observaciones. Hibiscus trionum es la única especie del género presente en la Flora Argentina con hábito herbáceo. 


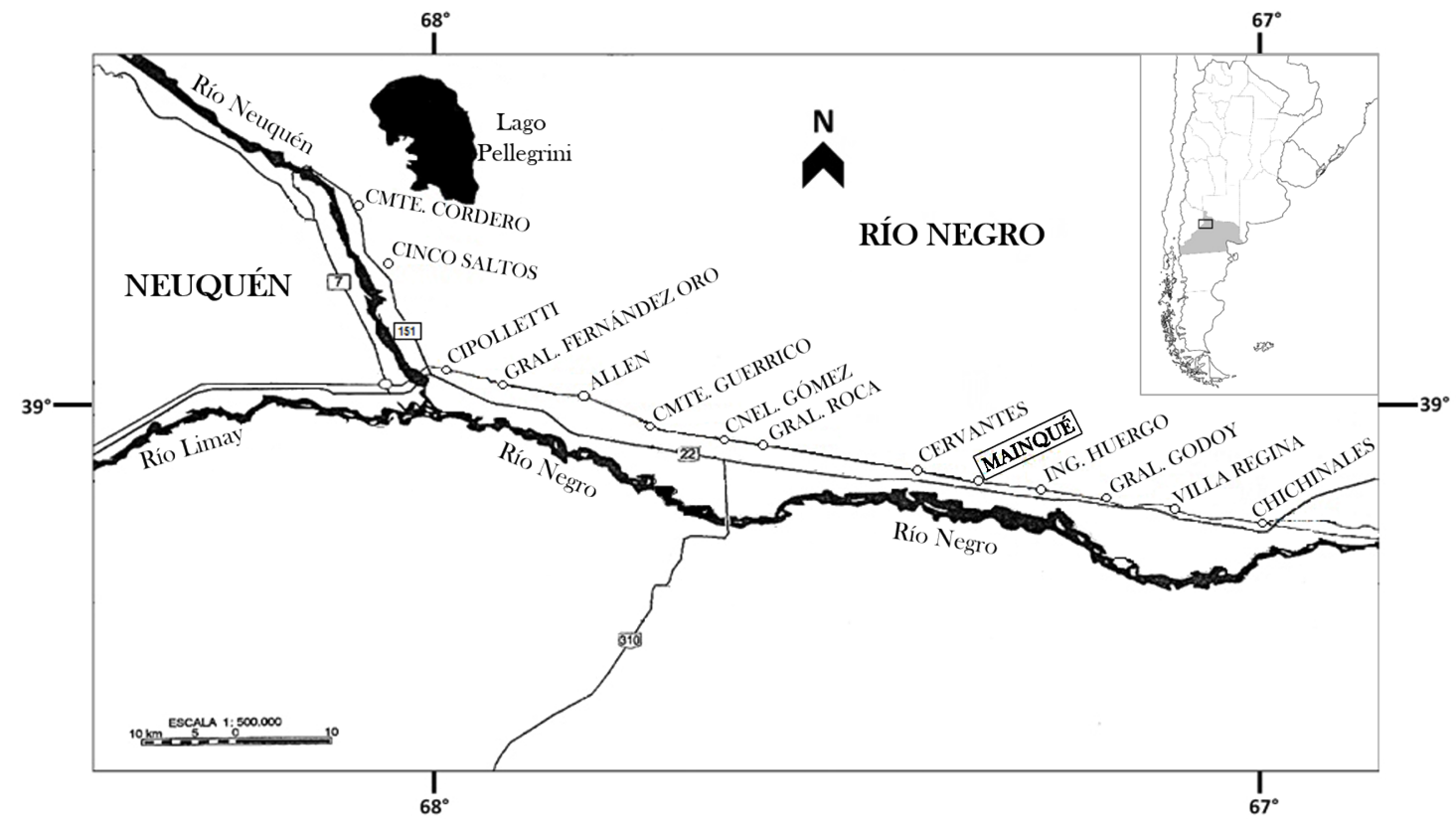

Fig. 3. Mapa del Alto Valle de Río Negro. La localidad de Mainqué fue el sitio de recolección de Hibiscus trionum L.

Se realizó un seguimiento de las poblaciones halladas durante tres temporadas consecutivas, lo que permitió constatar la extensión de las mismas año a año. Las semillas germinan en la primera quincena de enero (temperatura media de enero para los últimos 10 años: $23,09^{\circ} \mathrm{C}$ ) y la plena floración se alcanza en la segunda quincena del mes de marzo (temperatura media de marzo para los últimos 10 años: $17,9^{\circ} \mathrm{C}$ ). Un ensayo de flotabilidad de las semillas indicó que el $90 \%$ del total se mantenían en superficie, esto sugiere que el agua de las acequias de riego representa un medio de dispersión de la especie (resultados confirmados por los autores). Se apreció que las poblaciones se desarrollan en suelos con alto contenido en arcilla. Asimismo, se observó que aquellas plantas situadas en la fila del cultivo (región menos intervenida por labores culturales) presentan mayor altura (ca. $40 \mathrm{~cm}$ ) con respecto a las ubicadas en la zona del interfilar (entre 10 y $15 \mathrm{~cm}$ ).

Se registró que las flores permanecen abiertas durante un día y son visitadas por insectos polinizadores como Apis mellifera $\mathrm{L}$. (Hymenoptera, Apidae) y Bombus terrestris L.
(Hymenoptera, Apidae), que son atraídos, según Vignolini et al. (2015), por la iridiscencia existente en la región oscura de los pétalos. Se reconocieron daños causados por artrópodos como Tetranychus urticae C. L. Koch (Prostigmata, Tetranychidae) y Liriomyza sp. (Diptera, Agromyzidae).

Hibiscus trionum se caracteriza por tener una alta producción de semillas por fruto (entre 25 y 30 ), con una cubierta seminal resistente que le proporcionan un estado de latencia por varias temporadas en el suelo (Westra et al., 1996). Chachalis et al. (2008) demostraron que la germinación de semillas de $H$. trionum está influenciada por diversos efectos ambientales: las bajas temperaturas inducen el proceso de dormancia para evitar germinaciones en condiciones desfavorables; los mayores porcentajes de germinación ocurren con $\mathrm{pH}$ entre 7 y 8 ; la germinación y emergencia puede ocurrir aún bajo condiciones de estrés hídrico; una profundidad de siembra entre 0 y $2 \mathrm{~cm}$ generan mayores porcentajes de germinación, esto sugiere que la especie puede establecerse de manera adecuada en aquellos campos donde la labranza es nula o mínima. 
Los microorganismos fúngicos del suelo generan microrrupturas en la superficie de la semilla cuando se encuentra enterrada por un período de tiempo prolongado (alrededor de 8 meses), lo que promueve el ingreso de agua (Chachalis et al., 2008). Las condiciones óptimas de germinación, indicadas por estos últimos autores, son coincidentes con las del Alto Valle de Río Negro. Además, semillas provenientes del material recolectado, colocadas en un sustrato húmedo en caja de Petri a temperatura ambiente, demostraron una capacidad de germinación del $80 \%$ (resultados confirmados por los autores). A su vez, el uso de maquinaria agrícola y sus implementos (arado de disco) en suelos de texturas finas junto al agua para riego, distribuida a través de acequias, aumentaría la dispersión de la especie.
El establecimiento de densas poblaciones y las observaciones antes mencionadas confirman, según los criterios establecidos por Pyšek et al. (2004), la naturalización de H. trionum en el Alto Valle de Río Negro.

Se sospecha que la introducción de Hibiscus trionum al Alto Valle de Río Negro se originó a través del guano de gallinas empleado como enmienda, proveniente de la provincia de Buenos Aires (entrevistas con productores de la zona). Por este motivo no se descarta la presencia de $H$. trionum en otras regiones del país.

\section{Material representativo examinado}

ARGENTINA. Río Negro. Depto. General Roca, Mainqué, 23-III-2017 (fl, fr), Gandullo et al. 2051 (ARC); Mainqué, 15-III-2019 (fl, fr), Minué \& Gandullo 2182 (ARC).

\section{Clave para la identificación de las especies de Hibiscus en Argentina}

1. Plantas herbáceas

H. trionum

1. Plantas arbustivas

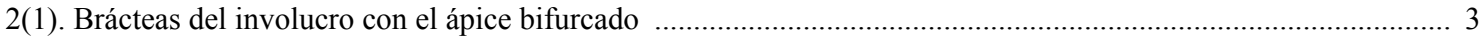

2. Brácteas del involucro con el ápice ensanchado o linear-acuminado ............................................................. 5

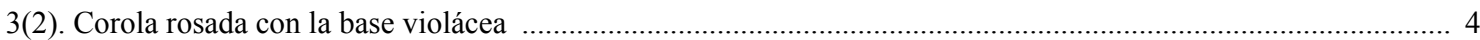

3. Corola morada H. radiatus

4(3). Tallo cubierto densamente con pelos estrellados, algunos simples H. furcellatus

4. Tallo subglabro, con pelos recurvos y aculéolos retrorsos H. bifurcatus

5(2). Hojas con la base marcadamente cordada, borde ligeramente crenado H. sororius

5. Hojas con la base obtusa, borde dentado o aserrado 6

6(5). Cápsula de 20-22 mm, brácteas del involucro de $20-25 \mathrm{~mm}$ H. urticifolium 6. Cápsula de 28-30 mm, brácteas del involucro de $15-18 \mathrm{~mm}$ H. striatus

\section{AGRADECIMIENTOS}

Los autores agradecen a los revisores anónimos de Darwiniana, nueva serie y a Adriel Jocou, por sus aportes y sugerencias; a Cristina Fernández y Viviana Troncoso por su colaboración durante los relevamientos realizados. El presente trabajo se financió con fondos del Proyecto PI A129 de la Secretaría de Ciencia y Técnica de la Universidad Nacional del Comahue.

\section{BIBLIOGRAFÍA}

CABI. 2019. Hibiscus trionum (Venice mallow), en Invasive Species Compendium. Wallingford, UK: CAB International. www.cabi.org/isc

Caius, J. F. 1942. The medicinal mallowworts of India. The Journal of the Bombay Natural History Society 43: 226-241.

Chachalis, D.; N. Korres \& E. M. Khah. 2008. Factors affecting seed germination and emergence of Venice mallow (Hibiscus trionum). Weed Science 56: 509-515. DOI: 10.1614/WS-07-144.1 


\section{R. MINUÉ \& R. GANDULLO. Hibiscus trionum en Argentina}

Cheers, G. 1999. Botanica, the illustrated A-Z of over 10,000 garden plants and how to cultivate them, $3^{\text {era }}$ ed. Milsons Point: Könemann.

Curtis, W. 1792. The botanical magazine; or flower-garden displayed, vol. 5-6, pp. 209.

Dimitri, M. J. 1972. Hibiscus, en M. J. Dimitri (dir.), Enciclopedia Argentina de Agricultura y Jardinería, $2^{\mathrm{da}}$ ed., vol. I, pp. 623-624. Buenos Aires: ACME.

Flora of Pakistan. 2019a. Malvaceae. Hibiscus. Disponible en: www.tropicos.org/Name/40025797?projectid=32 (Consulta: enero 2019).

Flora of Pakistan. 2019b. Hibiscus trionum. Disponible en: www.tropicos.org/Name/19600999? projectid=32 (Consulta: enero 2019).

Martínez Carretero, E. \& C. Ganci. 2011. Flora Urbana: Malvaceae Juss. Multequina 20: 1-6.

Mohlenbrock, R. H. (ed). 1931. The Illustrated Flora of Illinois: Flowering plants, basswoods to spurges. Carbondale \& Edwarsville: Southern Illinois University Press.

Naqshi, A. R.; G. H. Dar, G. N. Javeid \& P. Kachroo. 1988. Malvaceae of Jammu \& Kashmir State. Annals of the Missouri Botanical Garden 75: 1499-1524. DOI: 10.2307/2399298

Nogueira, I. \& J. Paiva. 2005. Hibiscus L., en S. Castroviejo, C. Aedo, M. Laínz, F. Muñoz Garmendia, G. Nieto Feliner, J. Paiva \& C. Benedí (eds.), Flora Ibérica 3: 196-199. Madrid: Real Jardín Botánico, CSIC.

Odero, D. C.; A. O. Mesbah, S. D. Miller \& A. R. Kniss. 2009. Venice Mallow (Hibiscus trionum) Interference in Sugarbeet. Weed Technology 23: 581-585. DOI: 10.1614/WT-09-045.1

Paiva, J. 2005. Malvaceae, en S. Castroviejo, C. Aedo, M. Laínz, F. Muñoz Garmendia, G. Nieto Feliner, J. Paiva \& C. Benedí. (eds.). Flora Ibérica 3: 190-191. Madrid: Real Jardín Botánico, CSIC.

Pammel, L. H. 1913. The weed flora of Iowa. Iowa Geological Survey, Bulletin $N^{\circ} 4$. Des Moines: Iowa Geological Survey.

Pyšek, P.; D. M. Richardson, M. Rejmánek, G. L. Webster, M. Williamson \& J. Kirschner. 2004. Alien plants in checklists and floras: towards better communication between taxonomists and ecologists. Taxon 53: 131-143. DOI: 10.2307/4135498

Rodrigo, A. del P. 1948. Los "Hibiscus" de la Flora Argentina (Malvaceae). Revista del Museo de La Plata, sección botánica 7: 111-154.

Rodríguez, R.; M. Baeza \& O. Matthei. 1989. Sobre la presencia de Hibiscus trionum L. (Malvaceae) en los cultivos de Chile. Gayana, Botánica 46: 117-120.
Sibthorp, J. \& J. E. Smith. 1830. Flora Graeca, sive, Plantarum rariorum historia, quas in provinciis aut insulis Graeciae, vol. 7. Londres: Typis Richardi Taylor et socii.

Sobrero, M. T.; S. Chaila, M. C. Ochoa \& M. G. Pece. 2014. Environmental requirements for the germination of Sphaeralcea bonariensis. Planta Daninha 32: 491-496. DOI: 10.1590/S0100-83582014000300004

Thiers, B. [permanentemente actualizado, consulta 2019] Index Herbariorum: a global directory of public herbaria and associated staff. New York Botanical Garden's Virtual Herbarium, http://sweetgum.nybg.org/science/ih

Tourn, S.; P. Diez de Ulzurrun, P. Platz \& R. Lasaga. 2018. Semillas de malezas en máquinas cosechadoras: presencia, abundancia y modo de eliminación. Instituto Nacional de Tecnología Agropecuaria: artículo de divulgación. Disponible en: https://inta. gob.ar/documentos/semillas-de-malezas-en-maquinarias-cosechadoras-presencia-abundancia-y-modo-de-eliminación

Vignolini, S.; E. Moyroud, T. Hingant, H. Banks, P. Rudall, U. Steiner \& B. Glover. 2015. The flower of Hibiscus trionum is both visibly and measurably iridescent. New Phytologist 205: 97-101. DOI: 10.1111/nph.12958

Walker, S.; H. Wu \& K. Bell. 2010. Emergence and Seed Persistence of Echinochloa colona, Urochloa panicoides and Hibiscus trionum in the Sub-tropical Environment of North-Eastern Australia. Plant Protection Quarterly 25: 127-132.

Westra, P.; C. H. Pearson \& R. Ristau. 1990. Control of Venice mallow (Hibiscus trionum) in corn (Zea mays) and onions (Allium cepa). Weed Technology 4: 500-504. DOI: 10.1017/ S0890037X00025859

Westra, P.; C. H. Pearson, R. Ristau \& F. Schweissing. 1996. Venice mallow (Hibiscus trionum) seed production and persistence in soil in Colorado. Weed Technology 10: 22-28. DOI: $10.1017 / \mathrm{S} 0890037 \mathrm{X} 00045656$

Winters, H. F. 1970. Our Hardy Hibiscus Species as Ornamentals. Economic Botany 24: 155-164. DOI: 10.1007/ BF02860595

Ya, T.; M. G. Gilbert \& L. J. Dorr. 2007. Malvaceae, Hibiscus trionum, en Z. Y. Wu, P. H. Raven \& D. Y. Hong (eds.), Flora of China 12: 293. Disponible en: eFloras.org (Consulta: febrero 2019).

Zuloaga, F.; O. Morrone \& M. J. Belgrano. [permanentemente actualizado, consulta 2019] Catálogo de las plantas del Cono Sur. Disponible en: http://www2.darwin.edu.ar/Proyectos/ FloraArgentina/fa.htm 